\title{
Finite groups determined by an inequality of the orders of their subgroups II
}

\author{
Marius Tărnăuceanu \\ October 25, 2016
}

\begin{abstract}
In this note we study a class of finite groups for which the orders of subgroups satisfy a certain inequality. In particular, characterizations of the well-known groups $\mathbb{Z}_{2} \times \mathbb{Z}_{2}$ and $S_{3}$ are obtained.
\end{abstract}

MSC2000 : Primary 20D60, 20D30; Secondary 20D15, 11A25.

Key words : finite groups, subgroup lattices, number of subgroups, arithmetic functions.

\section{Introduction}

Let $G$ be a finite group, $L(G)$ be the subgroup lattice of $G$ and

$$
\sigma_{1}(G)=\sum_{H \in L(G)} \frac{|H|}{|G|}=\sum_{H \in L(G)} \frac{1}{|G: H|} .
$$

The starting point for our discussion is given by the paper [1], where the groups $G$ with $\sigma_{1}(G) \leq 2$ have been determined. Recall also several basic properties of the function $\sigma_{1}$ :

- if $G$ is cyclic of order $n$ and $\sigma(n)$ denotes the sum of all divisors of $n$, then $\sigma_{1}(G)=\frac{\sigma(n)}{n}$;

- $\sigma_{1}$ is multiplicative, i.e. if $G_{i}, i=1,2, \ldots, m$, are finite groups of coprime orders, then $\sigma_{1}\left(\prod_{i=1}^{m} G_{i}\right)=\prod_{i=1}^{m} \sigma_{1}\left(G_{i}\right)$; 
- $\sigma_{1}(G) \geq \sigma_{1}(G / H), \forall H \unlhd G$.

By refining the proof of Theorem 1 of [1], in the current paper we will determine the finite groups $G$ satisfying $\sigma_{1}(G) \leq 2+\frac{4}{|G|}$. Our main result is the following.

Theorem 1. Let $G$ be a finite group of order $n$. Then:

a) $\sigma_{1}(G)<2+\frac{4}{n}$ if and only if $G$ is cyclic and $\sigma(n)<2 n+4$ or $G \cong$ $\mathbb{Z}_{2} \times \mathbb{Z}_{2}$

b) $\sigma_{1}(G)=2+\frac{4}{n}$ if and only if $G$ is cyclic and $\sigma(n)=2 n+4$ or $G \cong \mathbb{Z}_{3} \times \mathbb{Z}_{3}$ or $G \cong S_{3}$.

Two nice characterizations of $\mathbb{Z}_{2} \times \mathbb{Z}_{2}$ and $S_{3}$ can be inferred from the above theorem.

Corollary 2. $\mathbb{Z}_{2} \times \mathbb{Z}_{2}$ is the unique non-cyclic group $G$ satisfying $\sigma_{1}(G)<$ $2+\frac{4}{|G|}$, while $S_{3}$ is the unique non-abelian group $G$ satisfying $\sigma_{1}(G)=2+\frac{4}{|G|}$.

By Theorem 1 of [1], a finite group $G$ with $\sigma_{1}(G) \leq 2$ is cyclic of deficient or perfect order. Also, in Lemma 4 below we will show that a finite group $G$ with $\sigma_{1}(G)<2+\frac{4}{|G|}$ is always nilpotent. Inspired by these results, we came up with the following natural problem: is there a constant $c \in(2, \infty)$ such that if $\sigma_{1}(G)<c$ then $G$ is nilpotent? The answer to this problem is negative, as shows our next theorem.

Theorem 3. There are sequences of finite non-nilpotent groups $\left(G_{n}\right)_{n \in \mathbb{N}}$ such that $\sigma_{1}\left(G_{n}\right) \searrow 2$ for $n \rightarrow \infty$.

Finally, we note that an interesting open problem is whether there is a constant $c \in(2, \infty)$ such that if $\sigma_{1}(G)<c$ then $G$ is solvable.

Most of our notation is standard and will not be repeated here. Basic definitions and results on groups can be found in [3]. For subgroup lattice concepts we refer the reader to [2] and [4].

\section{Proof of the main results}

We start by proving two auxiliary results.

Lemma 4. Let $G$ be a finite group. If $\sigma_{1}(G)<2+\frac{4}{|G|}$ then $G$ is nilpotent, while if $\sigma_{1}(G)=2+\frac{4}{|G|}$ then $G$ is nilpotent or $G \cong S_{3}$. 
Proof. Assume that $\sigma_{1}(G)<2+\frac{4}{|G|}$ and $G$ is not nilpotent, that is it contains a non-normal maximal subgroup $M$. Then $M$ coincides with its normalizer in $G$ and so it has exactly $r=|G: M|$ conjugates, say $M_{1}, M_{2}, \ldots, M_{r}$. On the other hand, $L(G)$ cannot consist only of $1, G$ and $M_{1}, M_{2}, \ldots, M_{r}$. Therefore there is $N \leq G$ such that $N \neq 1, G, M_{1}, M_{2}, \ldots, M_{r}$. Since

$$
2+\frac{4}{|G|}>\sigma_{1}(G) \geq \frac{1+r|M|+|N|+|G|}{|G|}=2+\frac{|N|+1}{|G|},
$$

it follows that $N$ is a normal subgroup of order 2 and $L(G)=\left\{1, G, M_{1}, M_{2}, \ldots\right.$, $\left.M_{r}, N\right\}$. Then either $N \subset M_{i}, \forall i=1,2, \ldots, r$, or $N \cap M_{i}=1, \forall i=1,2, \ldots, r$. We infer that $G$ is either a 2-group or a cyclic group of order $2 p$ for some odd prime $p$, a contradiction.

Assume now that $\sigma_{1}(G)=2+\frac{4}{|G|}$ and $G$ is not nilpotent. Then, under the above notation, we must have $|N|=3$ and thus $G$ is a group of order $3 q$ for some prime $q$. Clearly, the conditions $r \mid 3$ and $r \equiv 1(\bmod q)$ imply $r=3$ and $q=2$. Hence $G \cong S_{3}$, completing the proof.

Lemma 5. Let $G$ be a non-cyclic p-group. If $\sigma_{1}(G)<2+\frac{4}{|G|}$ then $G \cong$ $\mathbb{Z}_{2} \times \mathbb{Z}_{2}$, while if $\sigma_{1}(G)=2+\frac{4}{|G|}$ then $G \cong \mathbb{Z}_{3} \times \mathbb{Z}_{3}$.

Proof. Let $|G|=p^{n}$ and $G / \Phi(G) \cong \mathbb{Z}_{p}^{k}$. Then $2 \leq k \leq n$. Assume that $\sigma_{1}(G) \leq 2+\frac{4}{p^{n}}$ and denote by $a_{k, p}(i)$ the number of subgroups of order $p^{i}$ in $\mathbb{Z}_{p}^{k}, i=0,1, \ldots, k$. If $k \geq 3$ then

$$
\begin{aligned}
\sigma_{1}(G) & \geq \frac{1}{p^{n}}\left(a_{k, p}(0) p^{n-k}+a_{k, p}(1) p^{n-k+1}+a_{k, p}(k-1) p^{n-1}+a_{k, p}(k) p^{n}\right) \\
& =\frac{1}{p^{k}}\left(a_{k, p}(0)+a_{k, p}(1) p+a_{k, p}(k-1) p^{k-1}+a_{k, p}(k) p^{k}\right) \\
& =\frac{1}{p^{k}}\left(1+\frac{p^{k}-1}{p-1} p+\frac{p^{k}-1}{p-1} p^{k-1}+p^{k}\right) \\
& >\frac{1}{p^{k}}\left(1+4 p^{k}\right)>4>2+\frac{4}{p^{n}}
\end{aligned}
$$

contradicting our hypothesis. Consequently, $k=2$. For $n>2$ we infer that

$$
2+\frac{4}{p^{n}} \geq \sigma_{1}(G) \geq \frac{1+(p+1) p^{n-1}+p^{n-2}+p^{n}}{p^{n}}
$$


which leads to

$$
4 \geq p^{n-1}+p^{n-2}+1
$$

a contradiction. Therefore $n=2$, i.e. $G \cong \mathbb{Z}_{p} \times \mathbb{Z}_{p}$. Moreover, we have

$$
2+\frac{4}{p^{2}} \geq \sigma_{1}(G)=\frac{1+(p+1) p+p^{2}}{p^{2}} \Longleftrightarrow 4 \geq p+1 \Longleftrightarrow p \in\{2,3\} .
$$

Thus $G \cong \mathbb{Z}_{2} \times \mathbb{Z}_{2}$ for $\sigma_{1}(G)<2+\frac{4}{p^{2}}$ and $G \cong \mathbb{Z}_{3} \times \mathbb{Z}_{3}$ for $\sigma_{1}(G)=2+\frac{4}{p^{2}}$, as desired.

We are now able to prove our main results.

Proof of Theorem 1. a) Assume that $\sigma_{1}(G)<2+\frac{4}{n}$. Then $G$ is nilpotent by Lemma 4 , which implies that it can be written as a direct product of its Sylow $p_{i}$-subgroups:

$$
G \cong \prod_{i=1}^{m} G_{i}
$$

For every $i=1,2, \ldots, m$, we have

$$
\sigma_{1}\left(G_{i}\right) \leq \sigma_{1}(G)<2+\frac{4}{n} \leq 2+\frac{4}{\left|G_{i}\right|} .
$$

By Lemma 5 it follows that either $G_{i}$ is cyclic or $G_{i} \cong \mathbb{Z}_{2} \times \mathbb{Z}_{2}$. We infer that either $G$ is cyclic or $G \cong \mathbb{Z}_{2} \times \mathbb{Z}_{2} \times \mathbb{Z}_{n^{\prime}}$ for some odd positive integer $n^{\prime}$. Suppose that $G$ is not cyclic. If $n^{\prime}>1$ we obtain

$$
2+\frac{1}{n^{\prime}}=2+\frac{4}{n}>\sigma_{1}(G)=\sigma_{1}\left(\mathbb{Z}_{2} \times \mathbb{Z}_{2}\right) \sigma_{1}\left(\mathbb{Z}_{n^{\prime}}\right)=\frac{11 \sigma\left(n^{\prime}\right)}{4 n^{\prime}},
$$

or equivalently

$$
11 \sigma\left(n^{\prime}\right)<8 n^{\prime}+4
$$

a contradiction. Thus $n^{\prime}=1$ and $G \cong \mathbb{Z}_{2} \times \mathbb{Z}_{2}$.

b) Assume that $\sigma_{1}(G)=2+\frac{4}{n}$ and $G \approx S_{3}$. Then $G$ is nilpotent by Lemma 4 and so it has a direct decomposition of type $(*)$. For $m \geq 2$ we have

$$
\sigma_{1}\left(G_{i}\right) \leq \sigma_{1}(G)=2+\frac{4}{n}<2+\frac{4}{\left|G_{i}\right|}, i=1,2, \ldots, m,
$$


and again every $G_{i}$ is cyclic or isomorphic to $\mathbb{Z}_{2} \times \mathbb{Z}_{2}$ by Lemma 5 . Therefore either $G$ is cyclic or $G \cong \mathbb{Z}_{2} \times \mathbb{Z}_{2} \times \mathbb{Z}_{n^{\prime}}$ for some odd positive integer $n^{\prime}$. In the second case the condition $\sigma_{1}(G)=2+\frac{4}{n}$ leads to

$$
11 \sigma\left(n^{\prime}\right)=8 n^{\prime}+4
$$

a contradiction. For $m=1$ it follows that $G$ is a $p$-group, and consequently it is cyclic or isomorphic to $\mathbb{Z}_{3} \times \mathbb{Z}_{3}$ by Lemma 5 . This completes the proof.

Proof of Theorem 3. Let $\left(p_{n}\right)_{n \in \mathbb{N}}$ be the sequence of prime numbers. By Dirichlet's theorem we infer that for every $n \in \mathbb{N}$ there is a prime $q_{n}$ such that $p_{n} \mid q_{n}-1$. Let $G_{n}$ be the non-nilpotent group of order $p_{n} q_{n}$. This contains one subgroup of order $1, q_{n}$ subgroups of order $p_{n}$, one subgroup of order $q_{n}$, and one subgroup of order $p_{n} q_{n}$. Then

$$
\sigma_{1}\left(G_{n}\right)=\frac{1+p_{n} q_{n}+q_{n}+p_{n} q_{n}}{p_{n} q_{n}}=2+\frac{1+\frac{1}{q_{n}}}{p_{n}},
$$

and clearly $\sigma_{1}\left(G_{n}\right) \searrow 2$ for $n \rightarrow \infty$, as desired.

\section{References}

[1] T. De Medts and M. Tărnăuceanu, Finite groups determined by an inequality of the orders of their subgroups, Bull. Belg. Math. Soc. Simon Stevin 15 (2008), 699-704.

[2] R. Schmidt, Subgroup lattices of groups, de Gruyter Expositions in Mathematics 14, de Gruyter, Berlin, 1994.

[3] M. Suzuki, Group theory, I, II, Springer-Verlag, Berlin, 1982, 1986.

[4] M. Tărnăuceanu, Groups determined by posets of subgroups, Ed. Matrix Rom, Bucureşti, 2006.

Marius Tărnăuceanu

Faculty of Mathematics

"Al.I. Cuza" University

Iaşi, Romania

e-mail: tarnauc@uaic.ro 